Bolyai Society - Springer-Verlag

\title{
SHADOWS AND ISOPERIMETRY UNDER THE SEQUENCE-SUBSEQUENCE RELATION
}

\author{
RUDOLF AHLSWEDE and NING CAI
}

Received May 26, 1995

Revised August 23, 1996

\section{Introduction and results}

One of the basic results in extremal set theory was discovered in [1] and rediscovered in [2]: For a given number of $k$-element subsets of an $n$-set the shadow, that is, the set of $(k-1)$-element subsets contained in at least one of the specified $k$-element subsets, is minimal, if the $k$-element subsets are chosen as an initial segment in the squashed order (see [10]; called colex order in [11]), that is, a $k$ element subset $A$ precedes a $k$-element subset $B$, if the largest element in $A \triangle B$ is in $B$. A closely related result was discovered in [3] and rediscovered in [5]: For a given number $u \in\left[0,2^{n}\right]$ of arbitrary subsets of an $n$-set the "Hamming distance 1"-boundary is minimal for the initial segment of size $u$, also called in short " $u$-th initial segment", in the $H$-order (of [3]), that is, if one chooses all subsets of cardinality less than $n-k$ ( $k$ suitable) and all remaining subsets of cardinality $n-k$, whose complements are in the initial segment of the squashed order. subsets.

In this paper we consider sequences and subsequences rather than sets and

The basic objects are $\mathscr{X}^{n}=\prod^{n} \mathscr{X}$ for $\mathscr{X}=\{0,1\}$ and $n \in \mathbb{N}$, and operations of deletion $\nabla_{i}, \nabla$ and of insertion $\triangle_{i}, \triangle$. Here $\nabla_{i}$ (resp. $\triangle_{i}$ ) means that letter $i$ $(i=0,1)$ is deleted (resp. inserted) and $\nabla$ (resp. $\triangle$ ) means the deletion (resp. insertion) of any letter.

So for $A \subset \mathscr{X}^{n}$ we get the down shadow

$$
\nabla A=\left\{x^{n-1} \in \mathscr{X}^{n-1}: x^{n-1} \text { is subsequence of some } a^{n} \in A\right\}
$$

and the up shadow

$$
\triangle A=\left\{x^{n+1} \in \mathscr{X}^{n+1}: \text { some subsequence of } x^{n+1} \text { is in } A\right\} .
$$

Mathematics Subject Classification (1991): 02 A 20, 05 D 05, 68 R 15 
In other words, $\nabla A$ are all sequences of length $n-1$ obtained by omitting any letter in the sequences of $A$. Then $\nabla_{i} A$ are all those sequences obtained by omitting the letter $i$. Clearly,

$$
\nabla A=\nabla_{0} A \cup \nabla_{1} A
$$

and analogously

$$
\triangle A=\triangle_{0} A \cup \triangle_{1} A
$$

We describe now our results.

\section{A. Shadows for fixed level and specific letter}

The $\ell$-th level is the set of sequences (or words)

$$
\mathscr{X}_{\ell}^{n}=\left\{x^{n} \in \mathscr{X}^{n}: \sum_{t=1}^{n} x_{t}=\ell\right\} .
$$

We consider sets $B \subset X_{n-k}^{n}$ of cardinality $v, 0 \leq v \leq\left(\begin{array}{l}n \\ k\end{array}\right)$, and their shadows $\nabla_{0} B, \triangle_{1} B$ (the other shadows can be estimated similarily by symmetry).

The unique binomial representation of $v$ is

$$
v=\left(\begin{array}{c}
a_{k} \\
k
\end{array}\right)+\left(\begin{array}{c}
a_{k-1} \\
k-1
\end{array}\right)+\ldots+\left(\begin{array}{c}
a_{s} \\
s
\end{array}\right)
$$

(with $a_{k}>a_{k-1}>\ldots>a_{s} \geq s \geq 1$ ).

Whereas Katona used in [2] and also in [6] the function $F$ :

$$
F(k, v)=\left(\begin{array}{c}
a_{k} \\
k-1
\end{array}\right)+\left(\begin{array}{c}
a_{k-1} \\
k-2
\end{array}\right)+\ldots+\left(\begin{array}{c}
a_{s} \\
s-1
\end{array}\right)
$$

we introduce and need here the functions $\stackrel{\nabla}{F}$ and $\stackrel{\Delta}{F}$, which play the analogue roles for the new shadow problems:

$$
\stackrel{\nabla}{F}(k, v)=\left(\begin{array}{c}
a_{k}-1 \\
k-1
\end{array}\right)+\left(\begin{array}{c}
a_{k-1}-1 \\
k-2
\end{array}\right)+\ldots+\left(\begin{array}{c}
a_{s}-1 \\
s-1
\end{array}\right)
$$

and

$$
\stackrel{\triangle}{F}(k, v)=\left(\begin{array}{c}
a_{k}+1 \\
k
\end{array}\right)+\left(\begin{array}{c}
a_{k-1}+1 \\
k-1
\end{array}\right)+\ldots+\left(\begin{array}{c}
a_{s}+1 \\
s
\end{array}\right)
$$


Theorem 1. For all $B \subset X_{n-k}^{n}$ with $|B|=v$

(i) $\left|\nabla_{0} B\right| \geq \stackrel{\nabla}{F}(k, v)$,

(ii) $\left|\triangle_{1} B\right| \geq \stackrel{\Delta}{F}(k, v)$,

and

(iii) both bounds are optimal.

\section{B. Shadows of arbitrary sets under deletion of any letter}

For any integer $u \in\left[0,2^{n}\right]$ we use the unique binomial representation

$$
u=\left(\begin{array}{l}
n \\
n
\end{array}\right)+\ldots+\left(\begin{array}{c}
n \\
k+1
\end{array}\right)+\left(\begin{array}{c}
\alpha_{k} \\
k
\end{array}\right)+\ldots+\left(\begin{array}{c}
\alpha_{t} \\
t
\end{array}\right)
$$

(with $n>\alpha_{k}>\ldots>\alpha_{t} \geq t \geq 1$ ) and observe that for an initial $H$-order segment $S$ with $|S|=u$

$$
|\nabla S|=\left(\begin{array}{c}
n-1 \\
n-1
\end{array}\right)+\left(\begin{array}{c}
n-1 \\
n-2
\end{array}\right)+\ldots+\left(\begin{array}{c}
n-1 \\
k
\end{array}\right)+\left(\begin{array}{c}
\alpha_{k}-1 \\
k-1
\end{array}\right)+\ldots+\left(\begin{array}{c}
\alpha_{t}-1 \\
t-1
\end{array}\right)
$$

$$
=\stackrel{\nabla}{G}(n, u), \text { say }
$$

Theorem 2. For every $A \subset \mathscr{X}^{n},|\nabla A| \geq \stackrel{\nabla}{G}(n,|A|)$ and the bound is achieved by the $|A|$-th initial segment in $H$-order.

This result was first obtained by D. E. Daykin and T. N. Danh [8]. We are grateful to David for his dramatic story about the complexity of their (first) proof. It gave us the impetus to (quickly) find a proof with fairly lengthy calculations with binomial coefficients. Subsequently Daykin-Danh gave also another proof, which can be found in the collection [9]. Then we gave a very "short proof" in [9] based on Lemma 6 of [6] and our inequality (2.5) below. Unfortunately, as was kindly pointed out by David, the original proof of (2.5) has an error in equation (6) of [9].

\section{Shadows of arbitrary sets under insertion of any letter}

For $u$ in the representation (1.10) we define

$$
\stackrel{\Delta}{G}(n, u)=\left(\begin{array}{c}
n+1 \\
n+1
\end{array}\right)+\left(\begin{array}{c}
n+1 \\
n
\end{array}\right)+\ldots+\left(\begin{array}{l}
n+1 \\
k+1
\end{array}\right)+\left(\begin{array}{c}
\alpha_{k}+1 \\
k
\end{array}\right)+\ldots+\left(\begin{array}{c}
\alpha_{t}+1 \\
t
\end{array}\right)
$$


Theorem 3. *For every $A \subset X^{n},|\triangle A| \geq \widehat{G}(n,|A|)$, and the bound is achieved by the $|A|$-th initial segment in $H$-order.

\section{Remarks.}

1. It must be emphasized that the $H$-order minimizes simultaneously both, the lower and the upper shadows. There is no such phenomenon in the Boolean lattice for "Kruskal-Katona"-type shadows. It has immediate consequences for isoperimetric problems.

2. Theorem 1 can be derived easily from Theorems 2 and 3 like Kruskal-Katona's Theorem from Harper's Theorem.

\section{Two isoperimetric inequalities}

It has been emphasized in [7] that isoperimetric inequalities in discrete metric spaces are fundamental principles in combinatorics. The goal is to minimize the union of a specified number of spheres of constant radius. We speak of an isoperimetric inequality, if this minimum is assumed for a set of sphere-centers, which themselves form a sphere (or quasi-sphere, if numbers do not permit a sphere).

For any $A \subset X^{*}=\bigcup_{n=0}^{\infty} X^{n}$ and any distance $d$ we define (the union of spheres of radius $r$ )

$$
\Gamma_{d}^{r}(A)=\left\{x^{n^{\prime}} \in X^{*}: d\left(x^{n^{\prime}}, a^{n}\right) \leq r \text { for some } a^{n} \in A\right\} .
$$

A prototype of a discrete isoperimetric inequality is the one discovered in [3], rediscovered in [5], and proved again in [6]. Here $d$ equals the Hamming distance $d_{H}$ and is defined on $\mathscr{X}^{n} \times \mathscr{X}^{n}$.

We recall the result. For

$$
G(n, u)=\left(\begin{array}{l}
n \\
n
\end{array}\right)+\left(\begin{array}{c}
n \\
n-1
\end{array}\right)+\ldots+\left(\begin{array}{l}
n \\
k
\end{array}\right)+\left(\begin{array}{c}
\alpha_{k} \\
k-1
\end{array}\right)+\ldots+\left(\begin{array}{c}
\alpha_{t} \\
t-1
\end{array}\right)
$$

and any $A \subset \mathscr{X}^{n}$

$$
\left|\Gamma_{d_{H}}^{r}(A)\right| \geq G(n,|A|)
$$

and the bound is achieved by the $|A|$-th initial segment in $H$-order (this is a sphere of radius $k$, if $\left.|A|=\sum_{j=0}^{k}\left(\begin{array}{l}n \\ j\end{array}\right)\right)$.

* A referee kindly pointed out to us that the equivalence of Theorem 2 and Theorem 3 can be derived with a theorem in "Variational principle in discrete extremal problems" by Bezrukov (Reihe Informatik Bericht tr-ri-94-152, Universität-GH-Paderborn). 
We define now two distances, $\theta$ and $\delta$, in $X^{*}$. For $x^{m}, y^{m^{\prime}} \in X^{*} \theta\left(x^{m}, y^{m^{\prime}}\right)$ counts the minimal number of insertions and deletions which transform one word into the other. $\delta\left(x^{m}, y^{m^{\prime}}\right)$ counts the minimal number of operations, if also exchanges of letters are allowed. Thus $\delta\left(x^{m}, y^{m^{\prime}}\right) \leq \theta\left(x^{m}, y^{m^{\prime}}\right)$.

Now observe that from (1.15) and our Theorems 2 and 3, we get immediately two inequalities.

Corollary 1. For $A \subset X^{n}$

(i) $\Gamma_{\theta}^{1}(A) \leq \stackrel{\nabla}{G}(n,|A|)+\stackrel{\triangle}{G}(n,|A|)$,

(ii) $\Gamma_{\delta}^{1}(A) \leq \stackrel{\nabla}{G}(n,|A|)+\stackrel{\Delta}{G}(n,|A|)+G(n,|A|)$,

and both bounds are achieved by the $|A|$-th initial segment in $H$-order.

Moreover, in Theorem 4 of Section 6 we have established those inequalities for every radius $r$. The exact formulation and the proof require a technical setup.

\section{Auxiliary results}

\section{A. Numerical inequalities}

While working on [7] Gyula Katona drew attention to the approach of EckhoffWegner [4] to prove Kruskal-Katona via the following inequality for $F$, defined in (1.7).

Lemma 1 (see [4]). For $k>1, v \leq v_{0}+v_{1}$,

$$
F(k, v) \leq \max \left(v_{0}, F\left(k, v_{1}\right)\right)+F\left(k-1, v_{0}\right)
$$

In fact, he used this idea also in his proof of the isoperimetric inequality for the Hamming space. He just had to establish the corresponding inequality for $G$, defined in (1.14).

Lemma 2 (Lemma 6 of [6]). If $0 \leq u_{1} \leq u_{0}$ and $u \leq u_{0}+u_{1}$, then

$$
G(n, u) \leq \max \left(u_{0}, G\left(n-1, u_{1}\right)\right)+G\left(n-1, u_{0}\right) .
$$

The discoveries in the present paper are similar inequalities for $\stackrel{\nabla}{F}, \stackrel{\Delta}{F}, \stackrel{\nabla}{G}$, and $\overleftrightarrow{G}$ (defined in (1.8), (1.9), (1.11), and (1.12)), which for cardinalities of shadows resp. boundaries considered describe their values for segments in the $H$-order.

We state first the inequalities for $F$. They are proved in the same way as those for $G$ below. 
$\stackrel{\nabla}{F}$-inequality: For $k>1$, if $v \leq v_{0}+v_{1}$ and $v_{0}<\stackrel{\nabla}{F}(k, v)$, then

$$
\stackrel{\nabla}{F}(k, v) \leq \stackrel{\nabla}{F}\left(k, v_{1}\right)+\stackrel{\nabla}{F}\left(k-1, v_{0}\right)
$$

$\vec{F}$-inequality: For $k>1$, if $v \leq v_{0}+v_{1}$, then

$$
\overleftrightarrow{F}(k, v) \leq \max \left(v_{0}+v_{1}, \stackrel{\vec{F}}{ }\left(k, v_{1}\right)\right)+\stackrel{\triangle}{F}\left(k-1, v_{0}\right) .
$$

Next we derive the inequalities for $G$.

$\stackrel{\nabla}{G}$-inequality: If $w_{1} \leq w_{0}<\stackrel{\nabla}{G}(n, w)$ and $w \leq w_{0}+w_{1}$, then

$$
\stackrel{\nabla}{G}(n, w) \leq \stackrel{\nabla}{G}\left(n-1, w_{0}\right)+\stackrel{\nabla}{G}\left(n-1, w_{1}\right) .
$$

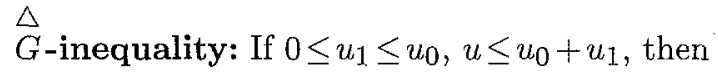

$$
\overleftrightarrow{G}(n, u) \leq \max \left(u_{0}+u_{1}, \overleftrightarrow{G}\left(n-1, u_{1}\right)\right)+\stackrel{\Delta}{G}\left(n-1, u_{0}\right) .
$$

Proofs. From the definitions of the numerical functions we have

$$
G(n, u)+u=\stackrel{\Delta}{G}(n, u) \text { for } u \text { as in }(1.10)
$$

and the equivalence of (2.2) and (2.6) immediately follows.

Next we show (2.5). For $u$ as in (1.10) denote by $\ell_{n}(u)$ and $r_{n}(u)$ the smallest $j$ with $\alpha_{j}>j$ and the number of $i$ 's with $\alpha_{i}=i$, respectively.

Let

$$
\begin{aligned}
\bar{u}(n-1) & \triangleq u-\stackrel{\nabla}{G}(n, u) \\
& =\left(\begin{array}{l}
n-1 \\
n-1
\end{array}\right)+\ldots+\left(\begin{array}{c}
n-1 \\
k+1
\end{array}\right)+\left(\begin{array}{c}
\alpha_{k}-1 \\
k
\end{array}\right)+\ldots+\left(\begin{array}{c}
\alpha_{\ell_{n}(u)}-1 \\
\ell_{n}(u)
\end{array}\right) .
\end{aligned}
$$

By (1.11) and (1.14)

$$
\begin{aligned}
& \underset{G}{\nabla}(n, u)=\left(\begin{array}{l}
n-1 \\
n-1
\end{array}\right)+\ldots+\left(\begin{array}{c}
n-1 \\
k
\end{array}\right)+\left(\begin{array}{c}
\alpha_{k}-1 \\
k-1
\end{array}\right)+\ldots+\left(\begin{array}{c}
\alpha_{\ell_{n}(u)}-1 \\
\ell_{n}(u)-1
\end{array}\right)+r_{n}(u) \\
& (2.8) \quad=G(n-1, \bar{u}(n-1))+r_{n}(u) .
\end{aligned}
$$

Moreover by the binomial coefficient representation

$$
u+1= \begin{cases}\left(\begin{array}{l}
n \\
n
\end{array}\right)+\ldots+\left(\begin{array}{c}
n \\
k+1
\end{array}\right)+\left(\begin{array}{c}
\alpha_{k} \\
k
\end{array}\right)+\ldots+\left(\begin{array}{c}
\ell_{n}(u) \\
\ell_{n}(u)-1
\end{array}\right) & \text { if } \alpha_{t}=t=1 \\
\left(\begin{array}{c}
n \\
n
\end{array}\right)+\ldots+\left(\begin{array}{c}
n \\
k+1
\end{array}\right)+\left(\begin{array}{c}
\alpha_{k} \\
k
\end{array}\right)+\ldots+\left(\begin{array}{c}
\alpha_{t} \\
t
\end{array}\right)+\left(\begin{array}{c}
t-1 \\
t-1
\end{array}\right) & \text { otherwise }\end{cases}
$$


(1.11) implies

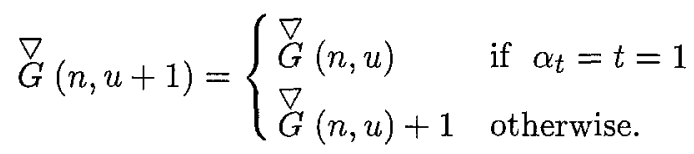

By the definition of binomial coefficient representation, $\bar{u}(n-1)$ in $(2.7)$ is non-decreasing in $u$ for fixed $n$ (c.f. $(2.9)$ ).

For $w_{1}, w_{0}$ and $w$ with

$$
w_{1} \leq w_{0}<\stackrel{\nabla}{G}(n, u)
$$

and

$$
w \leq w_{0}+w_{1}
$$

we let $w^{*}=w$, if $r_{n}(w)=0$, and otherwise

$$
\begin{aligned}
w^{*} & =\left(\begin{array}{l}
n \\
n
\end{array}\right)+\ldots+\left(\begin{array}{c}
n \\
k^{\prime}+1
\end{array}\right)+\left(\begin{array}{c}
\beta_{k^{\prime}} \\
k^{\prime}
\end{array}\right)+\ldots+\left(\begin{array}{c}
\beta_{\ell_{n}(w)} \\
\ell_{n}(w)
\end{array}\right)+\left(\begin{array}{c}
\ell_{n}(w) \\
\ell_{n}(w)-1
\end{array}\right) \\
(2.13) \quad & =w-r_{n}(w)+\ell_{n}(w),
\end{aligned}
$$

if the representation of $w$ is

$$
\begin{aligned}
w= & \left(\begin{array}{l}
n \\
n
\end{array}\right)+\ldots+\left(\begin{array}{c}
n \\
k^{\prime}+1
\end{array}\right)+\left(\begin{array}{c}
\beta_{k^{\prime}} \\
k^{\prime}
\end{array}\right)+\ldots+\left(\begin{array}{c}
\beta_{s} \\
s
\end{array}\right) \\
= & \left(\begin{array}{l}
n \\
n
\end{array}\right)+\ldots+\left(\begin{array}{c}
n \\
k^{\prime}+1
\end{array}\right)+\left(\begin{array}{c}
\beta_{k^{\prime}} \\
k^{\prime}
\end{array}\right)+\ldots+\left(\begin{array}{c}
\beta_{\ell_{n}(w)} \\
\ell_{n}(w)
\end{array}\right)+\left(\begin{array}{c}
\ell_{n}(w)-1 \\
\ell_{n}(w)-1
\end{array}\right)+ \\
& \ldots+\left(\begin{array}{c}
\ell_{n}(w)-r_{n}(w) \\
\ell_{n}(w)-r_{n}(w)
\end{array}\right) .
\end{aligned}
$$

\section{Write}

$$
w_{0}^{*}=w_{0}+\left(w^{*}-w\right) \text { and } w_{1}^{*}=w_{1} .
$$

Then by the definitions of $w^{*}, w_{0}^{*}, w_{1}^{*}$, and (2.10) (used repeatedly),

$$
\stackrel{\nabla}{G}\left(n, w^{*}\right)=\stackrel{\nabla}{G}(n, w)+\left(w^{*}-w\right)-1, \text { if } w^{*} \neq w
$$

and

$$
\stackrel{\nabla}{G}\left(n-1, w_{0}^{*}\right) \leq \stackrel{\nabla}{G}\left(n-1, w_{0}\right)+\left(w^{*}-w\right)-\tau\left(w_{0}^{*}\right)
$$


where $\tau\left(w_{0}^{*}\right)=1$, if $r_{n-1}\left(w_{0}^{*}\right)=0$ and $w_{0}^{*} \neq w_{0}$, and $\tau\left(w_{0}^{*}\right)=0$ otherwise. So, by (2.11), (2.14), and (2.16)

$$
w_{1}^{*} \leq w_{0}^{*} \leq \stackrel{\nabla}{G}\left(n, w^{*}\right),
$$

which with $(2.7),(2.8)$ and $(2.15)$ yields

$$
\begin{aligned}
\bar{w}_{0}^{*}(n-2)+G\left(n-2, \bar{w}_{0}^{*}(n-2)\right) & =w_{0}^{*}-\stackrel{\nabla}{G}\left(n-1, w_{0}^{*}\right)+G\left(n-2, \bar{w}_{0}^{*}(n-2)\right) \\
& =w_{0}^{*}-r_{n-1}\left(w_{0}^{*}\right) \leq w_{0}^{*} \leq \stackrel{\nabla}{G}\left(n, w^{*}\right) \\
& =G\left(n-1, \bar{w}^{*}(n-1)\right) .
\end{aligned}
$$

Moreover, by the first inequality in (2.18) and the monotonicity of $\bar{u}(n-1)$ (as a function of $u$ ),

$$
\bar{w}_{1}^{*}(n-2) \leq \bar{w}_{0}^{*}(n-2) .
$$

Now we assume that (2.5) does not hold and derive a contradiction. With (2.12) we obtain

$$
w-\stackrel{\nabla}{G}(n, w)<w_{0}-\stackrel{\nabla}{G}\left(n-1, w_{0}\right)+w_{1}-\stackrel{\nabla}{G}\left(n-1, w_{1}\right) .
$$

When $w^{*} \neq w$ then by (2.7) and (2.16) the LHS of $(2.22)$ is $w-\stackrel{\nabla}{G}\left(n, w^{*}\right)+$ $\left(w^{*}-w\right)-1=\bar{w}^{*}(n-1)-1$ and by (2.7), (2.14) and (2.17) the RHS of (2.22) is not bigger than $w_{0}-\stackrel{\nabla}{G}\left(n-1, w_{0}^{*}\right)+\left(w^{*}-w\right)-\tau\left(w_{0}^{*}\right)+\bar{w}_{1}^{*}(n-2) \leq \bar{w}_{0}^{*}(n-2)+\bar{w}_{1}^{*}(n-2)$.

Thus we have

$$
\bar{w}^{*}(n-1) \leq \bar{w}_{0}^{*}(n-2)+\bar{w}_{1}^{*}(n-2) .
$$

By our notation in (2.7), (2.21) certainly implies (2.22), when $w^{*}=w$ (so $\left.w_{0}^{*}=w_{0}\right)$.

Finally, with (2.19), (2.20), and (2.22) we obtain from Lemma 2,

$$
G\left(n-1, \bar{w}^{*}(n-1)\right) \leq G\left(n-2, \bar{w}_{0}^{*}(n-2)\right)+G\left(n-2, \bar{w}_{1}^{*}(n-2)\right) .
$$

This implies (2.5) (a contradiction to our assumption), because by (2.8), (2.15), and (2.16) the LHS of (2.23) is

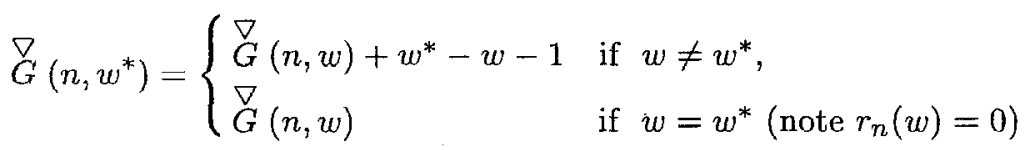


and by (2.8), (2.14), and (2.17) the RHS of (2.23) is

$$
\begin{gathered}
\stackrel{\nabla}{G}\left(n-1, w_{0}^{*}\right)+\stackrel{\nabla}{G}\left(n-1, w_{1}^{*}\right)-\left(r_{n-1}\left(w_{0}^{*}\right)+r_{n-1}\left(w_{1}^{*}\right)\right) \\
\leq \stackrel{\nabla}{G}\left(n-1, w_{0}\right)+\stackrel{\nabla}{G}\left(n-1, w_{1}\right)+w^{*}-w-\tau\left(w_{0}^{*}\right)-\left(r_{n-1}\left(w_{0}^{*}\right)+r_{n-1}\left(w_{1}^{*}\right)\right) \\
\leq \nabla / \nabla\left(n-1, w_{0}\right)+\stackrel{\nabla}{G}\left(n-1, w_{1}\right)+ \begin{cases}w^{*}-w-1 & \text { if } w \neq w^{*} \\
0 & \text { if } w=w^{*},\end{cases}
\end{gathered}
$$

\section{B. A calculus of iterative applications for $\stackrel{\nabla}{G}, \stackrel{\Delta}{G}$, and $G$}

We present here a rather technical result (Lemma 4 below), which is needed only for the proof of Theorem 4. Recall that for $u, 1 \leq u \leq 2^{n}$,

$$
\begin{aligned}
u & =\left(\begin{array}{l}
n \\
n
\end{array}\right)+\ldots+\left(\begin{array}{c}
n \\
k+1
\end{array}\right)+\left(\begin{array}{c}
\alpha_{k} \\
k
\end{array}\right)+\left(\begin{array}{c}
\alpha_{k-1} \\
k-1
\end{array}\right)+\ldots+\left(\begin{array}{c}
\alpha_{t} \\
t
\end{array}\right), \\
\stackrel{\nabla}{G}(n, u) & =\left(\begin{array}{l}
n-1 \\
n-1
\end{array}\right)+\ldots+\left(\begin{array}{l}
n-1 \\
k+1
\end{array}\right)+\left(\begin{array}{c}
n-1 \\
k
\end{array}\right)+\left(\begin{array}{c}
\alpha_{k}-1 \\
k-1
\end{array}\right)+\ldots+\left(\begin{array}{c}
\alpha_{t}-1 \\
t-1
\end{array}\right), \\
G(n, u) & =\left(\begin{array}{l}
n \\
n
\end{array}\right)+\ldots+\left(\begin{array}{c}
n \\
k+1
\end{array}\right)+\left(\begin{array}{l}
n \\
k
\end{array}\right)+\left(\begin{array}{c}
\alpha_{k} \\
k-1
\end{array}\right)+\ldots+\left(\begin{array}{c}
\alpha_{t} \\
t-1
\end{array}\right),
\end{aligned}
$$

and

$$
\vec{G}(n, u)=\left(\begin{array}{l}
n+1 \\
n+1
\end{array}\right)+\ldots+\left(\begin{array}{c}
n+1 \\
k+1
\end{array}\right)+\left(\begin{array}{c}
\alpha_{k}+1 \\
k
\end{array}\right)+\ldots+\left(\begin{array}{c}
\alpha_{t}-1 \\
t
\end{array}\right) .
$$

All these functions are increasing in $u$ and they transform binomial representations into binomial representations. This makes it easy to apply them repeatedly.

We notice that the representation of $\stackrel{\nabla}{G}(n, u)$ may be not unique, due to the appearance of the term $\left(\begin{array}{l}0 \\ 0\end{array}\right)$. However, it causes no difficulties to apply the functions, because both representations (if they exist) always give the same result, when $\underset{G}{G}$, $G$ or $\stackrel{\triangle}{G}$ are applied. More specifically, the non-uniqueness happens only when $\alpha_{t}=t=1$ in $(1.10)$, and with the notation $\ell_{n}(u)=\ell$ (say) in the proof of $(2.5)$,

$$
\begin{aligned}
\stackrel{\nabla}{G}(n, u)= & \left(\begin{array}{l}
n-1 \\
n-1
\end{array}\right)+\ldots+\left(\begin{array}{l}
n-1 \\
k+1
\end{array}\right)+\left(\begin{array}{c}
n-1 \\
k
\end{array}\right)+\left(\begin{array}{c}
\alpha_{k}-1 \\
k-1
\end{array}\right)+\ldots \\
& +\left(\begin{array}{c}
\alpha_{\ell}-1 \\
\ell-1
\end{array}\right)+\left(\begin{array}{l}
\ell-2 \\
\ell-2
\end{array}\right)+\ldots+\left(\begin{array}{l}
1 \\
1
\end{array}\right)+\left(\begin{array}{l}
0 \\
0
\end{array}\right)
\end{aligned}
$$




$$
=\left(\begin{array}{c}
n-1 \\
n-1
\end{array}\right)+\ldots+\left(\begin{array}{c}
n-1 \\
k
\end{array}\right)+\left(\begin{array}{c}
\alpha_{k}-1 \\
k-1
\end{array}\right)+\ldots+\left(\begin{array}{c}
\alpha_{\ell}-1 \\
\ell-1
\end{array}\right)+\left(\begin{array}{c}
\ell-1 \\
\ell-2
\end{array}\right) \triangleq v \text { say. }
$$

For the first representation of $v$

$$
\begin{aligned}
& \nabla \\
& G(n-1, v)= \\
& \left(\begin{array}{l}
n-2 \\
n-2
\end{array}\right)+\ldots+\left(\begin{array}{l}
n-2 \\
k-1
\end{array}\right)+\left(\begin{array}{c}
\alpha_{k}-2 \\
k-2
\end{array}\right)+\ldots+\left(\begin{array}{c}
\alpha_{\ell}-2 \\
\ell-2
\end{array}\right)+\left(\begin{array}{l}
\ell-3 \\
\ell-3
\end{array}\right)+\ldots+\left(\begin{array}{l}
0 \\
0
\end{array}\right), \\
& G(n-1, v)= \\
& \left(\begin{array}{l}
n-1 \\
n-1
\end{array}\right)+\ldots+\left(\begin{array}{l}
n-1 \\
k-1
\end{array}\right)+\left(\begin{array}{c}
\alpha_{k}-1 \\
k-2
\end{array}\right)+\ldots+\left(\begin{array}{c}
\alpha_{\ell}-1 \\
\ell-2
\end{array}\right)+\left(\begin{array}{l}
\ell-2 \\
\ell-3
\end{array}\right)+\ldots+\left(\begin{array}{l}
1 \\
0
\end{array}\right),
\end{aligned}
$$

and,

$\stackrel{\Delta}{G}(n-1, v)=\left(\begin{array}{l}n \\ n\end{array}\right)+\ldots+\left(\begin{array}{l}n \\ k\end{array}\right)+\left(\begin{array}{c}\alpha_{k} \\ k-1\end{array}\right)+\ldots+\left(\begin{array}{c}\alpha_{\ell} \\ \ell-1\end{array}\right)+\left(\begin{array}{l}\ell-1 \\ \ell-2\end{array}\right)+\ldots+\left(\begin{array}{l}2 \\ 1\end{array}\right)+\left(\begin{array}{l}1 \\ 0\end{array}\right)$,

and for the second representation of $v$,

$$
\begin{aligned}
& \stackrel{\nabla}{G}(n-1, v)=\left(\begin{array}{l}
n-2 \\
n-2
\end{array}\right)+\ldots+\left(\begin{array}{l}
n-2 \\
k-1
\end{array}\right)+\left(\begin{array}{c}
\alpha_{k}-2 \\
k-2
\end{array}\right)+\ldots+\left(\begin{array}{c}
\alpha_{\ell}-2 \\
\ell-2
\end{array}\right)+\left(\begin{array}{l}
\ell-2 \\
\ell-3
\end{array}\right), \\
& G(n-1, v)=\left(\begin{array}{l}
n-1 \\
n-1
\end{array}\right)+\ldots+\left(\begin{array}{l}
n-1 \\
k-1
\end{array}\right)+\left(\begin{array}{c}
\alpha_{k}-1 \\
k-2
\end{array}\right)+\ldots+\left(\begin{array}{c}
\alpha_{\ell}-1 \\
\ell-2
\end{array}\right)+\left(\begin{array}{l}
\ell-1 \\
\ell-3
\end{array}\right),
\end{aligned}
$$

and

$\vec{G}(n-1, v)=\left(\begin{array}{l}n \\ n\end{array}\right)+\ldots+\left(\begin{array}{l}n \\ k\end{array}\right)+\left(\begin{array}{c}\alpha_{k} \\ k-1\end{array}\right)+\ldots+\left(\begin{array}{c}\alpha_{\ell} \\ \ell-1\end{array}\right)+\left(\begin{array}{c}\ell \\ \ell-2\end{array}\right)$.

They really have the same values. define

For two functions $\phi, \psi: \mathbb{N} \rightarrow \mathbb{N}$ we write $\psi(\phi(\cdot))$ as $\psi \circ \phi(\cdot)$ and thus we can

$$
\begin{aligned}
& \stackrel{\nabla}{G}^{\circ p}(n, \cdot)=\stackrel{\nabla}{G}(n-p+1, \cdot) \circ \stackrel{\nabla}{G}(n-p+2, \cdot) \circ \ldots \circ \stackrel{\nabla}{G}(n, \cdot), \\
& G^{\circ q}(n, \cdot)=G(n, \cdot) \circ G(n, \cdot) \circ \ldots \circ G(n, \cdot),
\end{aligned}
$$

and

$$
\stackrel{\Delta}{G}^{\circ s}(n, \cdot)=\stackrel{\Delta}{G}(n+s-1, \cdot) \circ \stackrel{\Delta}{G}(n+s-2, \cdot) \circ \ldots \circ \stackrel{\Delta}{G}(n, \cdot)
$$

with $p, q$, and $s$ factors, respectively.

We can also define $\stackrel{\nabla}{G}^{o p}(n+s, \cdot) \circ \stackrel{\triangle}{G}^{o s}(n, \cdot), G^{\circ q_{\circ}} \stackrel{\Delta}{G}^{o p}$ etc.

Directly from the definitions the functions in $(2.24)-(2.26)$ can be calculated. 
Lemma 3. With the convention $\left(\begin{array}{l}k \\ \ell\end{array}\right)=0$ for $\ell<0$

$$
\begin{array}{r}
\stackrel{\nabla}{G}^{o p}(n, u)=\left(\begin{array}{l}
n-p \\
n-p
\end{array}\right)+\ldots+\left(\begin{array}{c}
n-p \\
k+1-p
\end{array}\right)+\left(\begin{array}{c}
\alpha_{k}-p \\
k-p
\end{array}\right)+\ldots+\left(\begin{array}{c}
\alpha_{t}-p \\
t-p
\end{array}\right) \\
G^{o q}(n, u)=\left(\begin{array}{l}
n \\
n
\end{array}\right)+\ldots+\left(\begin{array}{c}
n \\
k+1-q
\end{array}\right)+\left(\begin{array}{c}
\alpha_{k} \\
k-q
\end{array}\right)+\ldots+\left(\begin{array}{c}
\alpha_{t} \\
t-q
\end{array}\right)
\end{array}
$$

and

$$
\stackrel{\triangle}{G}^{o s}(n, u)=\left(\begin{array}{c}
n+s \\
n+s
\end{array}\right)+\ldots+\left(\begin{array}{c}
n+s \\
k+1
\end{array}\right)+\left(\begin{array}{c}
\alpha_{k}+s \\
k
\end{array}\right)+\ldots+\left(\begin{array}{c}
\alpha_{t}+s \\
t
\end{array}\right) .
$$

Here (2.28) is well-known from the isoperimetric theorem in the Hamming space.

Another important property of $G$-type functions is the commutativity of the o-operation:

$$
\stackrel{\nabla}{G} \circ G(n, u)=G \circ \stackrel{\nabla}{G}(n, u)=
$$

$$
\begin{aligned}
& \left(\begin{array}{l}
n-1 \\
n-1
\end{array}\right)+\ldots+\left(\begin{array}{c}
n-1 \\
k
\end{array}\right)+\left(\begin{array}{l}
n-1 \\
k-1
\end{array}\right)+\left(\begin{array}{c}
\alpha_{k}-1 \\
k-2
\end{array}\right)+\ldots+\left(\begin{array}{c}
\alpha_{t}-1 \\
t-2
\end{array}\right) \\
& \stackrel{\nabla}{G} \circ \stackrel{\Delta}{G}(n, u)=\stackrel{\vec{G}}{\nabla} \stackrel{\nabla}{G}(n, u)= \\
& \left(\begin{array}{l}
n \\
n
\end{array}\right)+\ldots+\left(\begin{array}{c}
n \\
k+1
\end{array}\right)+\left(\begin{array}{l}
n \\
k
\end{array}\right)+\left(\begin{array}{c}
\alpha_{k} \\
k-1
\end{array}\right)+\ldots+\left(\begin{array}{c}
\alpha_{t} \\
t-1
\end{array}\right)
\end{aligned}
$$

and

$$
G \circ \stackrel{\Delta}{G}(n, u)=\stackrel{\triangle}{G}_{\mathrm{G}} \circ G(n, u)=
$$

$$
\left(\begin{array}{c}
n+1 \\
n+1
\end{array}\right)+\ldots+\left(\begin{array}{c}
n+1 \\
k+1
\end{array}\right)+\left(\begin{array}{c}
n+1 \\
k
\end{array}\right)+\left(\begin{array}{c}
\alpha_{k}+1 \\
k-1
\end{array}\right)+\ldots+\left(\begin{array}{c}
\alpha_{t}+1 \\
t-1
\end{array}\right)
$$

Applying (2.27) - (2.29) and (2.30) - (2.32) repeatedly or by calculation we establish 'general rules.

Lemma 4. We have

$$
\begin{aligned}
& \stackrel{\nabla}{G}^{\circ p} \circ G^{\circ q} \circ \stackrel{\triangle}{G}^{o s}(n, u)=\stackrel{\nabla}{G}^{\circ p} \circ \stackrel{\Delta}{G}^{o s} \circ G^{\circ q}(n, u) \\
& =G^{\circ q_{\circ}} \stackrel{\nabla}{G}^{\circ p} \circ \stackrel{\triangle}{G}^{\circ S}(n, u)=G^{\circ q_{\circ}} \stackrel{\triangle}{G}^{\circ S} \circ \stackrel{\nabla}{G}^{\circ p} \cdot(n, u) \\
& =\stackrel{\triangle}{G}^{\circ s} \circ \stackrel{\nabla}{G}^{\circ p} \circ G^{\circ q}(n, u)=\stackrel{\triangle}{G}^{\circ S} \circ G^{\circ q} \circ \stackrel{\nabla}{G}^{\circ r}(n, u)
\end{aligned}
$$




$$
\begin{gathered}
=\left(\begin{array}{c}
n+s-p \\
n+s-p
\end{array}\right)+\left(\begin{array}{c}
n+s-p \\
n+s-p-1
\end{array}\right)+\ldots+\left(\begin{array}{c}
n+s-p \\
k+1-p-q
\end{array}\right) \\
+\left(\begin{array}{c}
\alpha_{k}+s-p \\
k-p-q
\end{array}\right)+\ldots+\left(\begin{array}{c}
\alpha_{t}+s-p \\
t-p-q
\end{array}\right)
\end{gathered}
$$

for $u$ as in $(1.10), 0 \leq p, q$, s.

\section{Proof of Theorem 1}

Denote an initial segment in squashed order (see [10]) over $X_{k}^{n}$ by $S$ and write $\bar{S}$ for the set of complements of the members of $S$. Thus $\bar{S} \subset X_{n-k}^{n}$ and $|\bar{S}|=|S|=v$, say. We speak here about the complementary squashed order or in short about the CS-order.

We consider first $\nabla_{0} \bar{S}$ and $\triangle_{1} \bar{S}$.

Lemma 5. For the initial segment $\bar{S}$ defined above

(i) $\nabla_{0} \bar{S}$ is the $\stackrel{\nabla}{F}(k, v)$-th initial segment in the CS-order on $X_{n-k}^{n-1}$ and

(ii) $\triangle_{1} \bar{S}$ is the $\stackrel{\triangle}{F}(k, v)$-th initial segment in the CS-order on $X_{n+1-k}^{n+1}$.

Proof. (i) We use the expansion (1.6) for $v$ and look at any $s^{n} \in \bar{S}$ :

$$
s_{t_{i}}=0 \text { for } i=1,2, \ldots, k \text { and } 1 \leq t_{1}<t_{2}<\ldots<t_{k} \leq n .
$$

By the definition of the CS-order there must be a $j$ such that for all $i \in(j, k]$ $t_{i}=a_{i}+1$ and for all $i \leq j t_{i} \leq a_{j}$. Now suppose that we delete for some index $\ell$ $s_{t_{\ell}}$. We can assume that $s_{t_{\ell}-1}=1$, because otherwise we can delete $S_{t_{\ell}-1}$ and get the same subsequence. Let $s^{\prime n-1}$ be the resulting subsequence, $t_{i}^{\prime}=t_{i}$ for $i<\ell$ and $t_{i-1}^{\prime}=t_{i}$ for $i>\ell$.

Choose now $j^{\prime}=\max (\ell, j)$ and notice that for $i \leq j^{\prime}-1, t_{i}^{\prime} \leq a_{j^{t}}-1$, for $i>j^{\prime}-1$ $t_{i-1}^{\prime}=a_{i}=\left(a_{i}-1\right)+1$, and for all $i s_{t_{i}^{\prime}}^{\prime}=0$.

Therefore the resulting subsequence $s^{n-1}$ falls into the $\underset{\nabla}{F}(k, v)$-th initial segment in CS-order.

Conversely, given a sequence $s^{\prime n-1}$ in $x_{n-k}^{n-1}$ and in the $\stackrel{\nabla}{F}(k, v)$-th initial segment the forgoing argument provides a way to find an $s^{n}$ in the $v$-th initial segment from which $s^{\prime n-1}$ is obtainable by deleting a 0 . 
(ii) Use again the $s^{n}$ described above and let $s^{\prime \prime n+1}$ be obtained by inserting a 1 before $s_{t_{\ell^{\prime \prime}}}, t_{i}^{\prime \prime}=t_{i}$ for $i<\ell^{\prime \prime}$ and $t_{i}^{\prime \prime}=t_{i}+1$ for $i \geq \ell^{\prime \prime}$.

Then $s_{t_{i}^{\prime \prime}}^{\prime \prime}=0$ for all $i$ and for $i \leq j^{\prime \prime} t_{i}^{\prime \prime} \leq a_{i}+1$; for $i>j^{\prime \prime} t_{i}^{\prime \prime}=a_{i}+2=\left(a_{i}+1\right)+1$, if we choose $j^{\prime \prime}=\max (j, \ell-1)$.

Clearly, such an $s^{\prime \prime n-1}$ is in the $\vec{F}(k, v)$-th initial segment in the CS-order. The same argument gives also the reverse implication.

Proof of Theorem 1 (i) and (ii) by induction on $n$.

The cases $n=1,2$ are done by simple inspection. For any $\ell, m, j, C \subset X^{\ell}$, $D \subset x^{m}$, and $E \subset X^{j}$ let

$$
\begin{aligned}
& C_{i}=\left\{\left(c_{1}, \ldots, c_{\ell-1}\right):\left(c_{1}, \ldots, c_{\ell-1}, i\right) \in C\right\}\left(\subset x^{\ell-1}\right), \\
& D * i=\left\{\left(d_{1}, \ldots, d_{m}, i\right):\left(d_{1}, \ldots, d_{m}\right) \in D\right\}\left(\subset x^{m+1}\right),
\end{aligned}
$$

and

$$
\hat{E}_{i}=\left\{\left(e_{1}, \ldots, e_{j}\right): e_{j}=i \text { and }\left(e_{1}, \ldots, e_{j}\right) \in E\right\}\left(\subset X^{j}\right)
$$

for $i=0,1$.

(i) for $n>2$.

Since $B_{0} \subset \nabla_{0} B,\left(\nabla_{0} B_{i}\right) * i \subset \nabla_{0} B(i=0,1)$ and $\left(\nabla_{0} B_{0}\right) * 0 \cap\left(\nabla_{0} B_{1}\right) * 1=\emptyset$, either $\left|\nabla_{0} B\right| \geq\left|B_{0}\right| \geq \stackrel{\nabla}{F}(k,|B|)$ or by $(2.3)$ and induction hypothesis $(\mathrm{IH})\left|\nabla_{0} B\right| \geq$ $\left|\nabla_{0} B_{0}\right|+\left|\nabla_{0} B_{1}\right| \stackrel{*}{\geq} \underset{F}{F}\left(k-1,\left|B_{0}\right|\right)+\stackrel{\nabla}{F}\left(k,\left|B_{1}\right|\right) \geq \stackrel{\nabla}{F}(k,|B|)$, where $(*)$ is justified by $B_{0} \subset X_{n-k}^{n-1}$, and $B_{1} \subset X_{n-k-1}^{n-1}$.

(ii) for $n>2$.

Recall the definition of the operator " $\wedge$ " in (3.3).

Considering $\triangle_{1} B=\left(\widehat{\triangle_{1} B_{1}}\right)_{1} \cup\left(\widehat{\triangle_{1} B}\right)_{0},\left(\widehat{\triangle_{1} B}\right)_{0}=\left(\triangle_{1} B_{0}\right) * 0, B * 1 \subset\left(\widehat{\triangle_{1} B}\right)_{1}$ and $\left(\triangle_{1} B_{1}\right) * 1 \subset\left(\widehat{\triangle_{1} B}\right)_{1}$, by $(2.4)$ and $\mathrm{IH}$,

$$
\begin{gathered}
\left|\triangle_{1} B\right| \geq \max \left(|B|,\left|\triangle_{1} B_{1}\right|\right)+\left|\triangle_{1} B_{0}\right| \geq \\
\max \left(|B|, \stackrel{\Delta}{F}\left(k,\left|B_{1}\right|\right)\right)+\stackrel{\Delta}{F}\left(k-1,\left|B_{0}\right|\right) \geq \vec{F}(k,|B|) .
\end{gathered}
$$

(iii) follows by Lemma 5 . 


\section{Proof of Theorem 2}

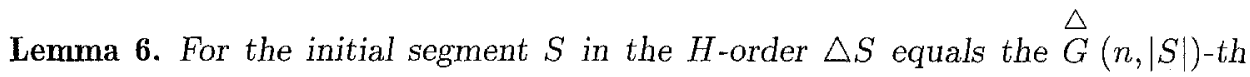
initial segment in the $H$-order, and $\nabla S$ equals the $\underset{\nabla}{\nabla}(n,|S|)$-th initial segment in $H$-order.

Proof. By the definitions of the two orders and direct inspection, we first get, that for some $k$ and $m$, and the $m$-th initial segment $S^{\prime}$ (of level $n-k$ ) in the CS-order

$$
\begin{aligned}
S & =\left(\bigcup_{\ell=0}^{n-(k+1)} x_{\ell}^{n}\right) \cup S^{\prime}, \\
\Delta S & =\left(\bigcup_{\ell=0}^{n-k} x_{\ell}^{n+1}\right) \cup \Delta_{1} S^{\prime},
\end{aligned}
$$

and

$$
\nabla S=\left(\bigcup_{\ell=0}^{n-(k+1)} x^{n-1}\right) \cup \nabla_{0} S^{\prime}
$$

The rest of the proof follows from Lemma 5 .

Proof of Theorem 2 by induction on $n$. For $n=2$ the statement is readily verified. From the IH for $n-1$ we proceed to $n$.

Next observe that, by convention (3.1) and (3.2), $\bigcup_{i=0}^{1}\left(\nabla A_{i}\right) * i \subset \nabla A, \bigcap_{i=0}^{1}\left(\nabla A_{i}\right) *$ $i=\emptyset$ and that therefore

$|\nabla A| \geq \sum_{i=0}^{1}\left|\nabla A_{i}\right| \geq \sum_{i=0}^{1} \nabla_{G}\left(n-1,\left|A_{i}\right|\right)$ (by the $\left.\mathrm{IH}\right)$.

According to the $\nabla$-inequality this can be lower bounded with the desired $\stackrel{\nabla}{G}(n,|A|)$, if $\left|A_{0}\right|,\left|A_{1}\right|<\stackrel{\nabla}{G}(n,|A|)$. Otherwise we have for some $i$ $\left|A_{i}\right|=\max \left(\left|A_{0}\right|,\left|A_{1}\right|\right) \geq \stackrel{\nabla}{G}(n,|A|)$ and we are done again, because $\nabla A \supset A_{i}$.

The achievability follows from Lemma 6. 


\section{Proof of Theorem 3}

The proof goes in exactly the same way as the proof of Theorem 1, (ii) (and the " $\triangle_{1}$ " part of (iii)), except that here we use (2.6), Lemma 6 and the observations: $\triangle A=(\widehat{\triangle A})_{1} \cup(\widehat{\triangle A})_{0},\left(\triangle A_{i}\right) * i \subset(\widehat{\triangle A})_{i}$ and $A * i \subset(\widehat{\triangle A})_{i}$ (for $i=0,1$ ).

\section{General isoperimetric theorems}

We use now the calculus of iterative applications of $\stackrel{\nabla}{G}, \stackrel{\Delta}{G}$, and $G$ described in Section 2 B.

Fortunately our Theorems 2, 3 and Harper's Theorem ([3]) establish the Inheritance property for the operations $\nabla, \triangle$, and $\Gamma_{d_{H}}^{1}$ (recall definition (1.13)). In the sequel, we abbreviate $\Gamma_{d_{H}}^{1}$ as $\Gamma_{d_{H}}$ and as $\Gamma$. If $S$ is an initial segment in $H$ order, then so are $\nabla S, \triangle S$, and $\Gamma_{d_{H}} S$. This enables us to apply these theorems repeatedly. Formally, we introduce

$$
\begin{aligned}
& \nabla^{\ell} A=\nabla(\nabla \ldots \nabla(\nabla A) \ldots), \\
& \triangle^{\ell} A=\triangle(\triangle \ldots \triangle(\triangle A) \ldots),
\end{aligned}
$$

and

$$
\begin{aligned}
\Gamma_{d_{H}}^{\ell} A & =\Gamma(\Gamma \ldots \Gamma(\Gamma A) \ldots) \\
& =\left\{x^{n} \in X^{n}: d_{H}\left(x^{n}, a^{n}\right) \leq \ell \text { for some: } a^{n} \in A\right\}
\end{aligned}
$$

and state the results.

Proposition 1. For every $A \subset \mathscr{X}^{n},|A|=u$

(i) $\left|\nabla^{\ell} A\right| \geq \nabla^{\circ \ell}(n, u)$

(ii) $\left|\triangle^{\ell} A\right| \geq \stackrel{\triangle}{G}^{\ell \ell}(n, u)$

(iii) $\left|\Gamma_{d_{H}}^{\ell} A\right| \geq G^{\circ \ell}(n, u)$

and all these bounds are achieved by the $u$-th initial segment in $H$-order.

Now we turn to the distances $\theta$ and $\delta$ in order to generalize Corollary 1 . Here operations are combined and the commutative law for the numerical functions (Lemma 4 in Section 2) is needed.

Fortunately this commutative law holds also for the operations $\nabla, \triangle$, and $\Gamma$ ! Indeed, using the short notation

$$
\nabla\left\{x^{n}\right\}=\nabla x^{n}, \triangle\left\{x^{n}\right\}=\triangle x^{n}, \Gamma\left\{x^{n}\right\}=\Gamma x^{n},
$$


we see that

$$
\nabla\left\{\triangle x^{n}\right\}=\triangle\left\{\nabla x^{n}\right\}, \Gamma\left\{\triangle x^{n}\right\}=\triangle\left\{\Gamma x^{n}\right\}, \nabla\left\{\Gamma x^{n}\right\}=\Gamma\left\{\nabla x^{n}\right\} .
$$

Therefore the commutative law holds for every $A \subset X^{n}$ :

$$
\nabla(\triangle A)=\triangle(\nabla A), \Gamma(\triangle A)=\triangle(\Gamma A), \nabla(\Gamma A)=\Gamma(\nabla A) .
$$

Moreover, it is clear that for every $A \subset \mathscr{X}^{n}$

$$
\Gamma^{\ell} A \subset \nabla^{\ell}\left(\triangle^{\ell} A\right)=\triangle^{\ell}\left(\nabla^{\ell} A\right) \text { for } \ell \leq n \text {. }
$$

Here strict inclusion can occur:

$$
\Gamma(1,0)=\{(0,0),(1,0),(1,1)\} \neq \mathscr{X}^{2}=\nabla(\Delta(1,0)) .
$$

However, strict inclusion does not occur, if $S$ is an initial segment in $H$-order.

Proposition 2. If $S$ is an initial segment in $H$-order, $|S|=u$, then

(i) $\left|\triangle^{\ell}\left(\nabla^{\ell} S\right)\right|=\left|\nabla^{\ell}\left(\triangle^{\ell} S\right)\right|=\stackrel{\triangle^{\ell \ell}}{{ }^{\ell}} \stackrel{\nabla^{\circ \ell}}{G}(n, u)=G^{\circ \ell}(n, u)=\left|\Gamma^{\ell} S\right|$

and

(ii) $\Delta^{\ell}\left(\nabla^{\ell} S\right)=\nabla^{\ell}\left(\Delta^{\ell} S\right) \equiv \Gamma^{\ell} S$

Proof. For (i) the first equalities are justified by (6.6) and Proposition 1 and the last equality is (the easy) part of Harper's Theorem. The remaining equality follows from Lemma 4 with the choices $p=s=\ell, q=0$ and $p=s=0, q=\ell$, respectively: both quantities equal $\left(\begin{array}{l}n \\ n\end{array}\right)+\ldots+\left(\begin{array}{c}n \\ k+1-\ell\end{array}\right)+\left(\begin{array}{c}\alpha_{k} \\ k-\ell\end{array}\right)+\ldots+\left(\begin{array}{c}\alpha_{t} \\ t-\ell\end{array}\right)$. Notice that (i) and (6.6) imply (ii).

Now we consider arbitrary sets $A \subset X^{n}$ and the distances $\theta, \delta$.

Proposition 3. For any $A \subset X^{n}, r>0$ and any $\ell_{i}, \ell_{i}^{\prime}(i=1,2)$ with $\ell_{2}-\ell_{1}=\ell_{2}^{\prime}-\ell_{1}^{\prime}$ and $\ell_{2}<\ell_{2}^{\prime}$

(i) $\nabla^{\ell_{2}}\left(\triangle^{\ell_{1}} A\right) \subset \nabla^{\ell_{2}^{\prime}}\left(\triangle^{\ell_{1}^{\prime}} A\right)$

and

(ii) $\Gamma_{\theta}^{r} A=\bigcup_{\ell=-r}^{r} \nabla^{\lfloor(r+\ell) / 2\rfloor}\left(\Delta^{\lfloor(r-\ell) / 2\rfloor} A\right)$

$$
=\bigcup_{\ell=0}^{r-1}\left[\left(\nabla^{\ell}\left(\Delta^{r-\ell} A\right)\right) \cup\left(\nabla^{\ell}\left(\Delta^{r-1-\ell} A\right)\right)\right] \cup \nabla^{r} A,
$$

where by convention $\triangle^{0} A=\nabla^{0} A=A$.

Proof. Obviously, for all $\ell$,

$$
A \subset \nabla^{\ell}\left(\triangle^{\ell} A\right)
$$


and therefore by the commutative law $(6.5)$

$$
\begin{aligned}
& \nabla^{\ell_{2}}\left(\triangle^{\ell_{1}} A\right) \subset \nabla^{\ell_{2}}\left(\triangle^{\ell_{1}}\left(\nabla^{\ell_{2}^{\prime}-\ell_{2}}\left(\triangle^{\ell_{2}^{\prime}-\ell_{2}} A\right)\right)\right)= \\
& \nabla^{\ell_{2}}\left(\triangle^{\ell_{1}}\left(\nabla^{\ell_{2}^{\prime}-\ell_{2}}\left(\triangle^{\ell_{1}^{\prime}-\ell_{1}} A\right)\right)=\nabla^{\ell_{2}^{\prime}}\left(\triangle^{\ell_{1}^{\prime}} A\right)\right),
\end{aligned}
$$

and thus (i) is verified.

Again by (6.5) and the definition of distance $\theta$

$$
\Gamma_{\theta}^{r} A=\bigcup_{r_{1}+r_{2} \leq r}\left(\nabla^{r_{2}}\left(\triangle^{r_{1}} A\right)\right)
$$

Thus by (i) and (6.9)

$$
\begin{aligned}
\Gamma_{\theta}^{r} & =\bigcup_{\ell=-r}^{r} \bigcup_{\substack{r_{1}+r_{2} \leq r \\
r_{2}-r_{1}=\ell}}\left(\nabla^{r_{2}}\left(\Delta^{r_{1}} A\right)\right)=\bigcup_{\ell=-r}^{r}\left(\nabla^{\lfloor(r+\ell) / 2\rfloor}\left(\Delta^{\lfloor(r-\ell) / 2\rfloor} A\right)\right) \\
& =\bigcup_{\ell=0}^{r-1}\left[\left(\nabla^{\ell}\left(\Delta^{r-\ell} A\right)\right) \cup\left(\nabla^{\ell}\left(\Delta^{r-1-\ell} A\right)\right)\right] \cup \nabla^{r} A .
\end{aligned}
$$

We are now ready to state and prove the main result.

Theorem 4. For all $A \subset \mathscr{X}^{n}$ and $r \geq 0$

(i) $\left|\Gamma_{\theta}^{r} A\right| \geq \sum_{\ell=-r}^{r} \nabla^{\circ\left\lfloor\frac{r+\ell}{2}\right\rfloor} \circ \Delta^{\left\lfloor\frac{r-\ell}{2}\right\rfloor}(n,|A|)$

and

(ii) $\left|\Gamma_{\delta}^{r} A\right| \geq \sum_{\ell=1}^{r}\left[\nabla^{\circ \ell} \circ G^{\mathrm{o}(r-\ell)}(n,|A|)+\stackrel{\triangle}{G}^{\circ \ell} \circ G^{\circ(r-\ell)}(n,|A|)\right]+G^{\mathrm{or}}(n,|A|)$,

where $G^{\circ 0}(n, u)=u$, and both bounds are achieved by the $|A|$-th initial segment in $H$-order.

Proof. By our definitions for $0 \leq \ell_{i}(i=1,2)$ and $n-\ell_{2}+\ell_{1} \geq 0$

$$
\nabla^{\ell_{2}}\left(\triangle^{\ell_{1}}\left(\Gamma^{\ell_{0}} A\right)\right) \subset \mathscr{X}^{n-\ell_{2}+\ell_{1}}
$$

(Here $\Gamma^{\ell_{0}}$ is only used for proving (ii).)

Therefore also

$$
\nabla^{\ell_{2}}\left(\triangle^{\ell_{1}}\left(\Gamma^{\ell_{0}} A\right)\right) \cap \nabla^{\ell_{2}^{\prime}}\left(\triangle^{\ell_{1}^{\prime}}\left(\Gamma^{\ell_{0}^{\prime}} A\right)\right)=\emptyset, \text { if } \ell_{1}-\ell_{2} \neq \ell_{1}^{\prime}-\ell_{2}^{\prime}
$$

and (i) as well as its optimality immediately follows from Proposition 3, (6.11) and Proposition 1 (applied twice). 
(ii) Similarly to (6.9), we have also

$$
\Gamma_{\delta}^{r} A=\bigcup_{r_{1}+r_{2}+r_{0} \leq r}\left(\nabla^{r_{2}}\left(\triangle^{r_{1}}\left(\Gamma_{d_{H}}^{r_{0}} A\right)\right)\right)
$$

and therefore

$$
\Gamma_{\delta}^{r} A \supset \bigcup_{\ell=1}^{r}\left(\nabla^{\ell}\left(\Gamma_{d_{H}}^{r-\ell} A\right)\right) \cup\left(\triangle^{\ell}\left(\Gamma_{d_{H}}^{r-\ell} A\right)\right) \cup \Gamma_{d_{H}}^{r} A
$$

Hence (ii) follows from (6.11), (6.13) and Proposition 1 (applied twice).

Finally, we have to show that the $|A|$-th initial segment in $H$-order $S$ achieves equality.

By Proposition 2 (ii), Proposition 3 (i), (6.12) and (6.11), and by the monotonicity of $\Delta^{t}, \nabla^{t}, \Gamma_{d_{H}}^{t}$ in the sets it suffices to show that for all parameters $-r \leq \ell \leq r, \ell_{1}+\ell_{2}+\ell_{0}=r, \ell_{2}-\ell_{1}=\ell$, and $\ell_{i} \geq 0$ for $i=0,1,2$

$$
\nabla^{\ell_{2}}\left(\triangle^{\ell_{1}}\left(\Gamma^{\ell_{0}} S\right)\right) \subset \begin{cases}\nabla^{\ell}\left(\Gamma^{r-\ell} S\right), & \text { if } \ell_{2}>\ell_{1} \\ \triangle^{|\ell|}\left(\Gamma^{r-|\ell|} S\right), & \text { if } \ell_{2}<\ell_{1} \\ \Gamma^{r} S, & \text { if } \ell_{2}=\ell_{1}\end{cases}
$$

Let us abbreviate $\nabla^{\ell_{2}}\left(\Delta^{\ell_{1}}\left(\Gamma^{\ell_{0}} S\right)\right)=L$.

Using Proposition 2 (ii) and Proposition 3 (i) we show the desired inclusions.

Case $\ell_{2}>\ell_{1}$.

$$
\begin{aligned}
L & =\nabla^{\ell_{2}-\ell_{1}}\left(\nabla^{\ell_{1}}\left(\triangle^{\ell_{1}}\left(\Gamma^{\ell_{0}} S\right)\right)\right) \\
& =\nabla^{\ell}\left(\Gamma^{\ell_{1}}\left(\Gamma^{\ell_{0}} S\right)\right)=\nabla^{\ell}\left(\Gamma^{\ell_{1}+\ell_{0}} S\right)=\nabla^{\ell}\left(\Gamma^{r-\ell_{2}} S\right) \\
& \subset \nabla^{\ell}\left(\Gamma^{r-\ell} S\right) \quad\left(\text { as } \ell_{2}>\ell_{1} \geq 0, r-\ell_{2} \leq r-\ell\right) .
\end{aligned}
$$

Case $\ell_{2}<\ell_{1}$.

$$
\begin{aligned}
L & =\Delta^{\ell_{1}-\ell_{2}}\left(\Delta^{\ell_{2}}\left(\nabla^{\ell_{2}}\left(\Gamma^{\ell_{0}} S\right)\right)\right) \\
& =\Delta^{|\ell|}\left(\Gamma^{\ell_{0}+\ell_{2}} S\right)=\Delta^{|\ell|}\left(\Gamma^{r-\ell_{1}} S\right) \\
& \subset \Delta^{|\ell|}\left(\Gamma^{r-|\ell|} S\right) \quad\left(\text { as } 0 \leq \ell_{2}<\ell_{1}, r-\ell_{1} \leq r+\ell=r-|\ell|\right),
\end{aligned}
$$

Case $\ell_{2}=\ell_{1}$.

$$
L=\nabla^{\ell_{1}}\left(\triangle^{\ell_{1}}\left(\Gamma^{\ell_{0}} S\right)\right)=\Gamma^{\ell_{0}+\ell_{1}} S \subset \Gamma^{r} S
$$




\section{References}

[1] J. B. KRUSKAL: The number of simplices in a complex, in: Mathematical optimization Techniques, Berkeley and Los Angeles, 1963, 251-278.

[2] G. Katona: A theorem on finite sets, in: Theory of Graphs Proc. Colloq. Tihany 1966, Akadémiai Kiadó, 1968, 187-207.

[3] L. H. HARPER: Optimal numberings and isoperimetric problems on graphs, $J$ : Combin. Theory, 1 (1966), 385-393.

[4] J. Eckhoff and G. Wegner: Über einen Satz von Kruskal, Periodica Math. Hungar., Vol 6 (2) (1975), 137-142.

[5] R. Ahlswede, P. GÁCS, and J. Körner: Bounds on conditional probabilities with applications in multiuser communication, 2. Wahrsch. und Verw. Gebiete, Vol. 34 (1976), 157-177.

[6] G. Katona: The Hamming sphere has minimum boundary, Studia Sci. Math. Hungar, 10 (1975), 131-140.

[7] R. Ahlswede and G. Katona: Contributions to the geometry of Hamming spaces, Discrete Math, 17 (1977), 1-22.

[8] D. E. DAYKIN: Oral communication.

[9] R. AHLSwEDE: Report on work in progress in combinatorial extremal theory: Shadows, AZ-identities, matching, SFB 343 Diskrete Strukturen in der Mathematik, Universität Bielefeld, Preprint (Ergänzungsreihe).

[10] I. Anderson: Combinatorics of Finite Sets, Clarendon Press, Oxford, 1987.

[11] B. Bollobás: Combinatorics, Cambridge University Press, 1986.

Rudolf Ahlswede

Universität Bielefeld

Fakultät für Mathematik

Postfach 100131

33501 Bielefeld

Germany

hollmann@mathematik.uni-bielefeld.de
Ning Cai

Universität Bielefeld

Fakultät für Mathematik

Postfach 100131

33501 Bielefeld

Germany

cai@mathematik.uni-bielefeld.de 